\title{
In-situ Monitoring TEM Specimen Preparation with Different Electron Detectors in a SEM-FIB System
}

Jian-Guo Zheng ${ }^{1}$

1. Irvine Materials Research Institute, University of California, Irvine, CA, USA.

Nowadays, SEM-FIB systems are commonly used for TEM specimen preparation because of their advantages, for example, specific site/orientation control and time saving, as compared with traditional techniques. Besides traditional Everhart-Thornley (E-T) "secondary electron" (SE) detector, various other electron detectors such as in-lens SE detector, in-lens backscattered electron (BSE) detector and STEM detector are available in the Tescan GAIA3 SEM-FIB system at UCI. These detectors are very useful for in-situ monitoring TEM specimen preparation. Here I would like to report some examples of applying these detectors in the final stage of the specimen preparation.

Tip sharpening process can be monitored by using a combination of in-lens SE detector and in-lens BSE detector. E-T detector and in-lens SE detector collect secondary electrons to show specimen morphology. In-lens SE images normally have a better resolution than E-T images. Figure 1 shows the in-lens SE image of a tip sample for TEM tomography. The tip is composed of top protection Pt layers, a thin film of interest indicated by an arrow, a $\mathrm{Si}(100)$ substrate. The tip is wielded on the top of an OmniProbe lift-out grid bar with ion-beam-induced Pt. The tip sharpening process can be monitored with the SE detector (Figures 1-3). However, it is difficult to see the difference between the protection $\mathrm{Pt}$ and Si substrate in the in-lens SE image when the tip becomes small (Figure 3). The in-lens BSE detector collects primary electrons to display specimen composition information, so the BSE image shows a strong contrast between $\mathrm{Pt}$ and $\mathrm{Si}$ (Figure 4). This is perfect to monitor how the protection $\mathrm{Pt}$ is depleting. We may stop the final tip polishing when the protection Pt almost disappears. With this method, tip samples with required tip diameters have been successfully prepared.

The Tescan GAIA3 SEM-FIB system has a 35-degree pretilt STEM holder mounted at the edge of the sample stage, which allows the SE image and STEM image to be recorded simultaneously (Figures 5-6). So cross-sectional TEM lamella can be monitored by using a combination of in-lens SE detector and STEM dark-field detector. Figures 5-8 show two pairs of TEM lamella images recorded with SE and STEM detectors, respectively. The white arrows indicate the interface (dark line) between the protection Pt (top) and the sample surface. Figure 5 and 7 displays sample surface morphology, while Figure 6 and 8 gives sample thickness information. We may monitor the depletion of the top Pt layer during the final polishing (Figures 5 and 7). Obviously, the STEM dark-field images (Figures 6 and 8) add another dimension to monitor thickness change of the region of interest (ROI) (the area just below the protection $\mathrm{Pt}$ ). When ROI is still thick, it is dark (Figure 6) because it is not electron transparent. When it becomes thinner (Figure 8), it is bright. If the STEM detector gain is fixed and the image intensity is calibrated, we may measure the sample thickness from the image intensity. In summary, the Tescan GAIA3 SEMFIB system has a unique capability to image the sample with both in-lens SE and STEM detectors at the same time. [1].

References:

[1] The author would acknowledge the use of IMRI facilities for the SEM/FIB work. 


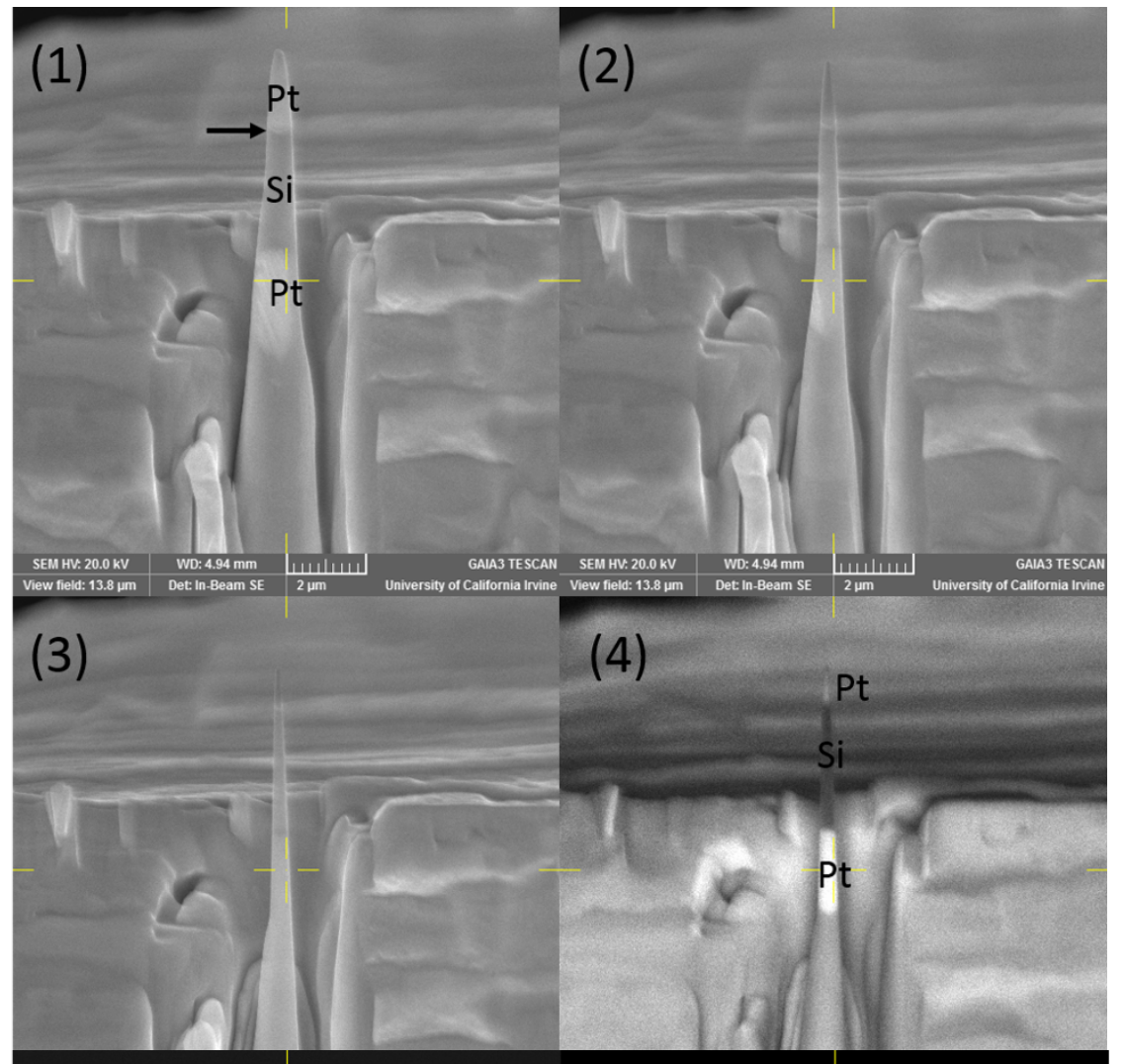

(5)

(6)

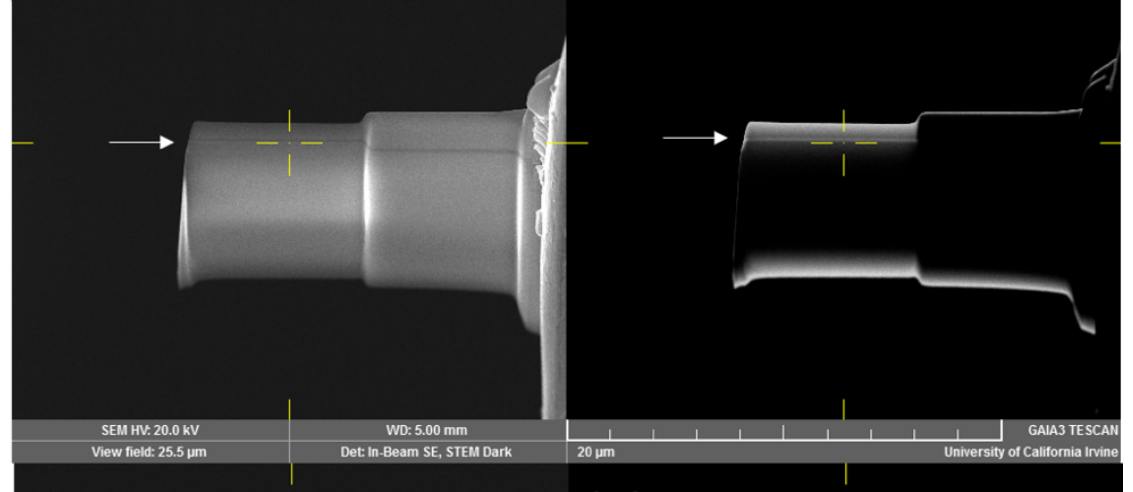

(7)

(8)

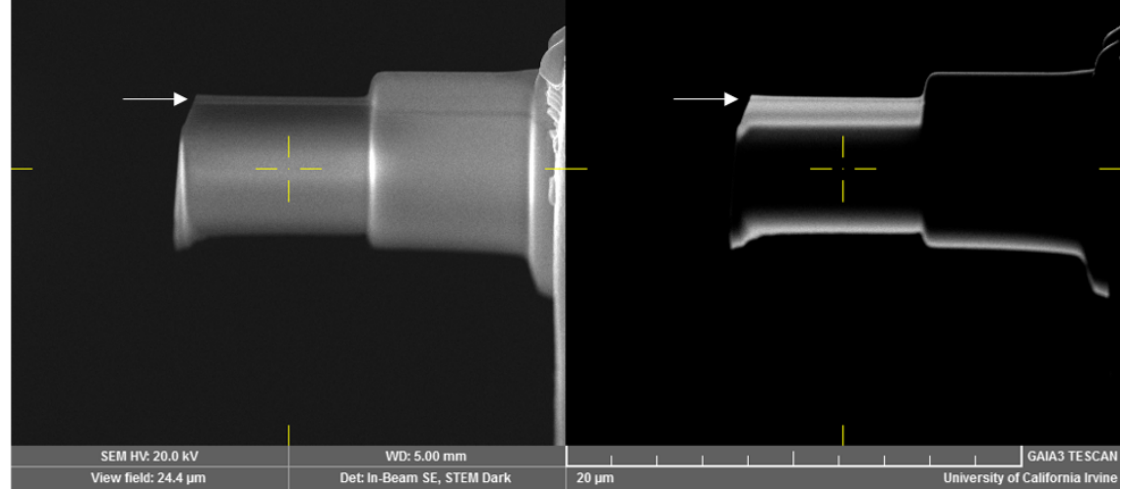

Figure 1. In-lens SE image of a test sample after initial tip shaping. The region of interest indicated by an arrow is protected by Pt layers (top) and grown on the $\mathrm{Si}$ (100) surface.

Figure 2. In-lens SE image of the tip sample after further sharpening by a $30 \mathrm{kV} \mathrm{Ga}$ ion beam with a circle pattern having an outside diameter of $2 \mu \mathrm{m}$ and inner diameter of $0.8 \mu \mathrm{m}$.

Figure 3. In-lens SE image of the tip sample after further sharpening by a $30 \mathrm{kV} \mathrm{Ga}$ ion beam with a circle pattern having an outside diameter of $0.8 \mu \mathrm{m}$ and inner diameter of $0.2 \mu \mathrm{m}$.

Figure 4. In-lens BSE image of the tip sample showed in Figure 3. The intensity of $\mathrm{Pt}$ area is much higher than Si area.

Figure 5. In-lens SE image of a cross-sectional TEM lamella after initial thinning using Gan ion beam at $30 \mathrm{kV}$ and $1 \mathrm{nA}$. The dark line is the interface between the protection $\mathrm{Pt}$ layers (top) and region of interest (ROI) grown on the Si (100).

Figure 6. STEM dark-field image recorded simultaneously with Figure 5 from the same sample. The RIO is dark (thick).

Figure 7. In-lens SE image of the cross-sectional TEM lamella after further thinning using Gan ion beam at $30 \mathrm{kV}$ and $200 \mathrm{pA}$. The top $P t$ is almost depleted.

Figure 8. STEM dark-field image recorded simultaneously with Figure 7 from the same sample. The RIO is bright (thin). 